\title{
Validação de definições para indicadores do resultado NOC: Autocontrole da doença cardíaca
}

Validation of definitions of the indicators for Nursing Outcomes Classification outcomes: Self-management cardiac disease Validación de definiciones para indicadores del resultado NOC: Autocontrol de la enfermedad cardíaca

\author{
Agueda Maria Ruiz Zimmer Cavalcante ib https://orcid.org/0000-0003-3910-2162 \\ Camila Takao Lopes io https://orcid.org/0000-0002-6243-6497? \\ Elizabeth Swanson io https://orcid.org/0000-0002-3208-5278 \\ Sue Ann Moorhead io https://orididorg/0000-0002-9517-9909 ${ }^{3}$ \\ Maria Márcia Bachion io https://orcid.org/0000-0001-5044-6148 ${ }^{1}$ \\ Alba Lucia Bottura Leite de Barros io hittps://orcid.org/0000-0003-2691-3492²
}

\section{Como citar:}

Cavalcante AM, Lopes CT, Swanson E, Moorhead AS, Bachion MM, Barros AL. Validação de definições para indicadores do resultado NOC: Autocontrole da doença cardíaca. Acta Paul Enferm. 2020;33:e-APE20180265

DOI

http://dx.doi.org/10.37689/ acta-ape/2020A00265

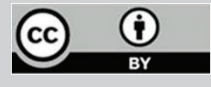

Descritores

Insuficiência cardíaca; Avaliacão de resultados da assistência ao paciente; Avaliação em Enfermagem; Estudos de validação

Keywords

Heart failure; Patient outcome assessment; Nursing assessment; Validation studies

Descriptores Insuficiencia cardíaca; Evaluación del resultado de la atención al paciente; Evaluación en enfermeira; Estudios de validación

Submetido 26 de Outubro de 2018

Aceito

25 de Julho de 2019

Autor correspondente Agueda Maria Ruiz Zimmer Cavalcante

E-mail: aguedamrzc@gmail.com https://orcid.org/0000-0003-3910-2162

\section{Resumo}

Objetivo: Validar definições conceituais e operacionais para os indicadores do resultado NOC "Autocontrole da doença cardíaca".

Métodos: Estudo metodológico de validação consensual desenvolvido em três etapas: revisão integrativa da literatura, elaboração de definições conceituais e operacionais para os indicadores do resultado NOC "Autocontrole da doença cardíaca" e validação das definições por consenso de 20 especialistas. Foi realizado teste binomial para análise da proporção de especialistas que concordaram que as definições elaboradas eram relevantes e claras. Valores de $p$ inferiores a 0,05 indicavam diferença significativa na opinião dos especialistas quanto à relevância e a clareza das definições.

Resultados: Na avaliação dos especialistas, as definições conceituais de 43 indicadores apresentaram valores de $p>0,05$ para clareza e 43 para relevância. Nas definições operacionais 36 indicadores apresentaram valor de $p>0,05$ para clareza e 43 para relevância. Para indicadores com $0 p<0,05$ reajustes foram feitos conforme as sugestões dos especialistas.

Conclusão: 0 estabelecimento de definições conceituais e operacionais para indicadores NOC torna 0 processo de avaliação mais confiável, orientando a prática clínica em direção a melhores resultados. Quando validados, esses indicadores podem oferecer maior precisão, aumentando a efetividade da prática clínica.

\section{Abstract}

Objective: To validate conceptual and operational definitions of the indicators for NOC outcomes: cardiac disease self-management.

Methods: This consensus-validation study was developed in three steps: integrative literature review, development of conceptual and operational definitions of the indicators for NOC outcome: cardiac disease self-management, and consensus-validation of definitions by 20 nursing specialists. A binomial test was conducted to analyze the proportion of nursing specialists who agreed on the relevance and clarity of definitions. P-values lower than 0.05 indicated a significant difference of the opinion among nursing specialits concerning the relevance and clarity of definitions.

Results: After the reviewing by nursing specialits, the conceptual definitions of 43 indicators for clarity and $43 \mathrm{for}$ relevance had a $p>0.05$. Operational definitions of 36 indicators presented for clarity and 43 for relevance had a $p$-value $>0.05$. Indicators showing $p<0.05$ were adjusted accordingly to reflect the opinion of nursing specialists. Conclusion: To establish conceptual and operational definitions for NOC indicators turn the assessment process more, and guide the clinical practice towards better results. Once validated, these indicators may provide higher precision and increase effectiveness in clinical practice. 


\section{Resumen}

Objetivo: Validar definiciones conceptuales y operativas para los indicadores del resultado NOC "Autocontrol de la enfermedad cardíaca"

Métodos: Estudio metodológico de validación consensual realizado en tres etapas: revisión integradora de la literatura, elaboración de definiciones conceptuales y operacionales para los indicadores del resultado NOC "Autocontrol de la enfermedad cardíaca" y validación de las definiciones por consenso de 20 especialistas. Se realizó test binomial para analizar la proporción de especialistas que estaba de acuerdo con que las definiciones elaboradas eran relevantes y claras. Valores de $p$ inferiores a 0,05 indicaban diferencia significativa en la opinión de los especialistas con relación a la relevancia y claridad de las definiciones.

Resultados: En el análisis de los especialistas, las definiciones conceptuales de 43 indicadores presentaron valores de $p>0,05$ respecto a la claridad y 43 a la relevancia. En las definiciones operativas, 36 indicadores presentaron valores de $p>0,05$ respecto a la claridad y 43 a la relevancia. Con relación a los indicadores con $p<0,05$, se realizaron ajustes según las sugerencias de los especialistas.

Conclusión: Establecer definiciones conceptuales y operativas para indicadores NOC permite que el proceso de evaluación sea más confiable, lo que orienta la práctica clínica a la obtención de mejores resultados. Al validarlos, estos indicadores pueden ofrecer mayor precisión y aumentar la efectividad de la práctica médica.

\section{Introdução}

Dentre os indicadores de qualidade do serviço prestado em instituiçóes de saúde, podem ser destacados os resultados apresentados pelo paciente, em relação ao seu estado de saúde ou qualidade de vida, decorrentes da assistência recebida. ${ }^{(1)}$ Eles configuram uma dimensão essencial para o estabelecimento de parâmetros de avaliaçáo, análise e tomada de decisão ${ }^{(1)}$ com vistas à melhora dos cuidados de saúde nos diversos contextos de atendimento. ${ }^{(2)}$

A definição de resultados esperados nas condiçóes de saúde do paciente e a verificaçáo de seu alcance ocorrem na terceira e na última etapas do Processo de Enfermagem (PE), ou seja, no planejamento e na avaliação da assistência implementada, respectivamente. Na fase de avaliaçáo, os enfermeiros analisam a efetividade das intervençôes implementadas, para orientar o replanejamento e fornecer informaçóes que permitem avaliar o resultado final da assistência. ${ }^{(3,4)}$

Para que se possa realizar a consolidaçáo e a gestão de informaçôes, é necessário utilizar linguagens padronizadas. Para descrever os resultados de enfermagem podem ser utilizadas classificaçóes, tais como a Nursing Outcomes Classification. ${ }^{(5)}$ Nessa Classificação, o resultado de enfermagem é definido como "estado ou comportamento do paciente, família ou comunidade sensível à intervenção de enfermagem". ${ }^{(5)}$ Cada resultado apresenta uma definição e um conjunto de indicadores. Estes, por sua vez, são definidos como "um estado, comportamento ou percepçóes observáveis ou avaliaçóes relatadas do paciente em nível mais concreto". ${ }^{(5)}$

Cada indicador é acompanhado por uma escala Likert de cinco pontos que consiste em opçôes para demonstrar a variabilidade no estado, comportamento ou percepçáo descrita pelo resultado. Os resultados NOC mostram como os pacientes respondem às intervençóes e ajudam a determinar se mudanças nos cuidados são necessárias. Os indicadores, no entanto, precisam ser refinados e ter definições que permitam aos avaliadores estimar de maneira confiável as mudanças nos pacientes. ${ }^{(6)}$

Quando bem delineados e claros, os indicadores contribuem no reconhecimento e coleta de informações, descrevendo o estado de um fenômeno, el ou orientando as ações de enfermagem. ${ }^{(7)}$ Tal clareza é desejada para aumentar a acurácia do uso de sistemas de classificação. ${ }^{(6,8)}$ Logo, os indicadores de resultados necessitam de descrição conceitual e operacional para que possam ser aplicáveis à prática clínica.

Estudos têm sido desenvolvidos em diferentes contextos da prática, visando validar os resultados NOC e torná-los mais confiáveis. ${ }^{(6-8,8,10)}$ proporcionando maior uniformidade na avaliação clínica dos pacientes na prática.

No contexto de pessoas com insuficiência cardíaca (IC), os resultados obtidos mediante os cuidados prestados pelos enfermeiros podem ser demonstrados pela melhora clínica do indivíduo e diminuição de episódios de agudização do quadro clínico, pela adesáo ao tratamento medicamentoso e não medicamentoso, e pela redução readmissōes hospitalares. ${ }^{(11)}$

A IC é uma das doenças crônicas não transmissíveis mais prevalentes no mundo, afetando, mais de 23 milhôes de pessoas. ${ }^{(12)}$ No Brasil, no ano de 2018, houve 1.151.050 internaçôes por doenças do aparelho circulatório, sendo 200.694 por IC e 22.328 indivíduos foram a óbito devido à IC. ${ }^{(13)}$ 
Apesar dos avanços no tratamento, os pacientes apresentam alto risco de hospitalização por exacerbação da doença levando a redução da qualidade de vida e aumento da mortalidade. ${ }^{(14)}$ As intervençóes de autocontrole objetivam melhorar o conhecimento e as habilidades dos pacientes para realizar o autocuidado, auxiliando-os na adesão ao tratamento, modificaçóes no estilo de vida, monitoramento de sinais e sintomas e, consequentemente, prevenção de exacerbaçôes. ${ }^{(15)}$

Os enfermeiros desempenham um papel importante na implementação de intervençóes que capacitam o paciente na adesão ao tratamento, na identificação de complicaçóes e sinais e sintomas de agravos e na tomada de decisão diante de situaçôes de emergência. ${ }^{(16)}$ Nesse panorama, programas de gerenciamento de doenças crônicas são conduzidos por diferentes instituiçôes, onde os enfermeiros acompanham os pacientes ensinando-os e empoderando-os ao controle da doença.

O resultado NOC "Autocontrole da Doença Cardíaca" - definido como "açóes pessoais para o controle da doença cardíaca, seu tratamento e prevenção da progressão da doença e complicaçôes" - é compatível com os elementos de tratamento dos programas de gerenciamento da doença crônica. Pertence ao domínio Conhecimento e Comportamento em Saúde e a classe Comportamento de Saúde e inclui 45 indicadores que náo foram previamente validados. ${ }^{(5)}$

Embora alguns estudos tenham sido realizados utilizando os resultados NOC no cuidado à pessoas com IC em ambulatórios ou em domicílio, "Autocontrole da doença cardíaca" foi mencionado apenas uma vez. ${ }^{(2,17-19)}$ Os indicadores desse resultado atendem às recomendaçóes nacionais e internacionais à essa clientela. ${ }^{(20-23)}$

Em uma análise mais criteriosa, os resultados mencionados nesses estudos - Conhecimento: controle da doença cardíaca, Comportamento de manutenção do peso, Conhecimento: medicação, Controle de sintomas, Equilíbrio hídrico, Tolerância à atividade e Conservação de energia estão compreendidos nos indicadores de "Autocontrole da doença cardíaca". Isso significa que uma avaliação adequada do estado de um paciente com IC pode ser obtida usando apenas esse resultado. ${ }^{(2,17-19)}$
Considerando a importância de avaliar e mensurar os resultados de saúde do paciente utilizando indicadores, bem como o uso de linguagens padronizadas, o objetivo deste estudo foi validar definiçôes conceituais e operacionais para os indicadores do resultado NOC "Autocontrole da doença cardíaca”.

\section{Métodos}

Trata-se de um estudo metodológico, de validação consensual de definiçóes conceituais e operacionais dos indicadores de "Autocontrole da doença cardíaca" realizado em três etapas: revisão da literatura, desenvolvimento de definiçôes conceituais e operacionais para os indicadores, juntamente com definição de magnitude operacional para cada escore na escala de avaliação, e, finalmente, a validação por especialistas. ${ }^{(24)}$

Foram consultadas as seguintes bases de dados para a revisão: Literatura Latino-Americana e do Caribe em Ciências da Saúde (Lilacs), Medical Literature Analysis and Retrieval System Online (Medline), Cumulative Index to Nursing and Allied Health Literature (CINAHL) and Cochrane Database of Systematic Reviews. Artigos publicados em inglês, português ou espanhol, de 2009-2015, abordando o autocuidado de indivíduos com IC como o principal fenômeno em estudo foram incluídos. A delimitação do período inicial de inclusão (ano de 2009) se justifica pelo interesse em estudos publicados após o desenvolvimento da teoria do autocuidado para pessoas com IC. ${ }^{(25)}$

Com base nessa revisão da literatura, ${ }^{(25)}$ foram elaboradas as definiçóes conceituais e operacionais dos indicadores, bem como definiçóes para cada um dos cinco pontos da escala de Likert.

Definiçốes conceituais relacionam-se à palavra em si, considerando uma síntese do conceito. É definida de acordo com o significado bruto da palavra. As definiçóes operacionais atribuem significado a uma determinada palavra, especificando como este conceito deverá ser mensurável ou avaliado. (26) $\mathrm{Na}$ presente investigação, as definiçóes conceituais e operacionais dos indicadores foram analisadas, quanto à sua relevância e clareza, por enfermeiros especialistas. 
Estes profissionais foram inicialmente selecionados da Plataforma Lattes do Conselho Nacional de Desenvolvimento Científico e Tecnológico (CNPq) (http://lattes.cnpq.br), utilizando o termo "resultado NOC" e, em seguida, por amostragem de bola de neve, aqueles que aceitaram participar do estudo também indicaram outros enfermeiros.

Foram considerados especialistas, aqueles que alcançaram pontuação igual ou maior a seis ao preencher os seguintes critérios: ter concluído doutorado na área de terminologia de enfermagem e/ou cardiologia (2 pontos), mestrado na área de terminologia de enfermagem e/ou cardiologia (2 pontos), especialista na área de enfermagem em cardiologia (2 pontos), apresentar artigos publicados resultantes de tese ou dissertação na área de terminologia de enfermagem e/ou cardiologia (2 pontos), artigos publicados na área de terminologia de enfermagem e/ou Cardiologia (2 pontos), experiência docente na área de terminologia e/ou Cardiologia (2 pontos). (23) Além disso, os enfermeiros deviam preencher pelo menos um destes critérios: experiência clínica em Cardiologia há pelo menos dois anos (2 pontos) ou atuação em ambulatório de educação em saúde (2 pontos). (27) Assim, nesse estudo, foi estabelecido o escore igual ou maior que oito pontos (ou quatro critérios preenchidos) para ser considerado especialista. $\mathrm{O}$ recrutamento de enfermeiros julgados potencialmente elegíveis ocorreu em setembro de 2014 e o processo de validação ocorreu em 2015.

Um total de 65 enfermeiros foi convidado para participar no estudo. Desses, 30 concordaram em participar, mas dois deles alcançaram menos de 8 pontos.

$\mathrm{O}$ instrumento para a avaliação das definiçóes conceituais e operacionais dos indicadores do resultado NOC foi enviado por e-mail para os 28 especialistas, dos quais 20 responderam. A pontuaçáo alcançada pelos especialistas nos critérios de elegibilidade variou de 10 a 16 pontos, sendo a média de $12,75 \pm 2,22$.

Para avaliar a relevância e a clareza das definiçóes conceituais e operacionais os especialistas pontuaram com -1 (não adequado), 0 (indecisões quanto à adequação) ou +1 (adequado). ${ }^{(28)} \mathrm{Da}$ mesma forma foi avaliada clareza e relevância para as definiçóes operacionais da magnitude dos escores da escala Likert utilizada.

A partir do valor atribuído foi realizado o teste binomial, que avaliou a proporção de especialistas que concordaram que as definiçôes elaboradas eram relevantes e claras. Foi considerado adequado quando a proporção foi $\geq 85 \%$. ${ }^{(24)}$

O valor de $\mathrm{p}<0,05$ foi utilizado para indicar significância estatística quando a proporção era inferior a $85 \%$. Definiçôes com valores de $\mathrm{p}$ inferiores foram revisadas de acordo com sugestóes de especialistas.

Foi obtida a aprovação junto ao comitê de ética em pesquisa com seres humanos da instituição cede do estudo sob o CAEE de número 42990214.4.0000.5505, e o Termo de Consentimento Livre e Esclarecido foi enviado aos participantes, garantindo-lhes confidencialidade nas informaçóes e anonimato.

\section{Resultados}

A revisão da literatura permitiu elaborar as definiçôes conceituais e operacionais para cada um dos 45 indicadores do resultado de Autocontrole da doença cardíaca, além da definição operacional para a magnitude dos respectivos escores.

A maioria das definiçóes foi considerada clara e relevante. Observa-se que clareza das definiçóes operacionais obteve valores inferiores aos atribuídos à relevância (Tabela 1).

Os especialistas fizeram sugestóes sobre várias definiçóes com o objetivo de melhorá-las e então foram realizados os ajustes para melhorar sua adequação.

Em relação às definições conceituais, foram revisadas quanto à clareza para o indicador "Monitora a frequência e o ritmo de pulsaçóes" e quanto à relevância e clareza para o indicador "Segue a dieta recomendada" (Tabela 1 ).

As definições operacionais foram revisadas quanto à clareza para os indicadores: "Aceita o diagnóstico de saúde do provedor de cuidados"; "Participa do programa prescrito de reabilitação cardíaca”; "Implementa o regime de tratamento prescrito"; 
Tabela 1. Frequência de concordância entre os especialistas quanto à relevância e clareza das definições conceituais e operacionais dos indicadores do resultado NOC "Autocontrole da doença cardíaca"

\begin{tabular}{|c|c|c|c|c|}
\hline \multirow{2}{*}{$\begin{array}{l}\text { Autocontrole da doença cardíaca } \\
\text { Indicadores }\end{array}$} & \multicolumn{2}{|c|}{$\begin{array}{c}\text { Definição } \\
\text { conceitual } \\
p \text {-value }\left(\% \%^{\star *}\right)\end{array}$} & \multicolumn{2}{|c|}{$\begin{array}{c}\text { Definiçãa } \\
\text { operacional } \\
p \text {-value }\left(\%{ }^{\star *}\right)\end{array}$} \\
\hline & Relevância & Clareza & Relevância & Clareza \\
\hline 161701 - Aceita o diagnóstico de saúde do provedor de cuidados & $0,82(90,0)$ & $0,17(75,0)$ & $0,60(85,0)$ & $0,02(65,0)$ \\
\hline 161702 - Busca informações sobre métodos para manter a saúde cardiovascular & $0,96(95,0)$ & $0,60(85,0)$ & $1,00(100,0)$ & $0,82(90,0)$ \\
\hline 161703 - Participa no processo de tomada de decisão sobre cuidados de saúde & $1,00(100,0)$ & $0,60(85,0)$ & $0,96(95,0)$ & $0,60(85,0)$ \\
\hline 161704 - Participa no programa prescrito de reabilitação cardíaca & $1,00(100,0)$ & $0,96(95 \%)$ & $0,35(80,0)$ & $0,00(50 \%)$ \\
\hline 161705 - Implementa o regime de tratamento prescrito & $0,96(95,0)$ & $0,60(85 \%)$ & $0,35(80,0)$ & $0,01(60 \%)$ \\
\hline 161706 - Monitora o surgimento dos sintomas & $0,96(95,0)$ & $0,82(90 \%)$ & $0,82(90,0)$ & $0,17(75,0)$ \\
\hline 161707 - Monitora a persistência dos sintomas & $0,82(90,0)$ & $0,82(90 \%)$ & $0,96(95,0)$ & $0,01(60,0)$ \\
\hline 161708 - Monitora a gravidade dos sintomas & $1,00(100,0)$ & $0,82(90,0)$ & $0,96(95,0)$ & $0,02(65,0)$ \\
\hline 161709 - Monitora a frequência dos sintomas & $0,96(95,0)$ & $0,96(95,0)$ & $0,82(90,0)$ & $0,35(82,0)$ \\
\hline 161710 - Relata sinais de piora da doença & $0,82(90,0)$ & $0,82(90,0)$ & $0,82(90,0)$ & $0,17(75,0)$ \\
\hline 161711 - Relata sinais e sintomas de depressão & $0,35(80,0)$ & $0,60(85,0)$ & $0,07(70,0)$ & $0,00(55,0)$ \\
\hline 161712- Usa um diário para monitorar os sintomas ao longo do tempo & $0,17(75,0)$ & $0,35(80,0)$ & $0,17(75,0)$ & $0,07(70,0)$ \\
\hline 161713 - Usa medidas preventivas para reduzir o risco de complicações & $0,82(90,0)$ & $0,60(85,0)$ & $0,96(95,0)$ & $0,96(95,0)$ \\
\hline 161714 - Usa medidas de alívio dos sintomas & $1,00(100,0)$ & $1,00(100,0)$ & $0,82(90,0)$ & $0,35(80,0)$ \\
\hline 161744 - Obtém cuidados de saúde diante de ocorrência de sinais de alerta & $0,82(90,0)$ & $0,35(80,0)$ & $0,82(90,0)$ & $0,60(85,0)$ \\
\hline 161716 - Monitora a frequência e o ritmo de pulsações & $0,35(80,0)$ & $0,02(65,0)$ & $0,35(80,0)$ & $0,00(50,0)$ \\
\hline 161717 - Monitora a pressão sanguínea & $0,96(95,0)$ & $0,35(80,0)$ & $0,82(90,0)$ & $0,07(70,0)$ \\
\hline 161718 - Limita a ingestão de sódio & $0,96(95,0)$ & $0,60(85,0)$ & $0,82(90,0)$ & $0,60(85,0)$ \\
\hline 161719 - Limita a ingestão de gordura e colesterol & $0,96(95,0)$ & $0,96(95,0)$ & $0,60(85,0)$ & $0,17(75,0)$ \\
\hline 161720 - Atende à dieta recomendada & $1,00(100,0)$ & $0,35(80,0)$ & $0,96(95,0)$ & $0,60(75,0)$ \\
\hline 161721 - Atende às recomendações de restrição de líquidos & $0,96(95,0)$ & $0,82(90,0)$ & $0,60(85,0)$ & $0,07(70,0)$ \\
\hline 161722 - Monitora os efeitos dos estimulantes & $0,60(85,0)$ & $0,07(70,0)$ & $0,96(95,0)$ & $0,35(80,0)$ \\
\hline 161723 - Monitora o peso do corpo & $0,96(95,0)$ & $0,82(90,0)$ & $0,82(90,0)$ & $0,82(90,0)$ \\
\hline 161724 - Usa estratégias eficazes de controle do peso & $0,96(95,0)$ & $0,35(80,0)$ & $0,96(95,0)$ & $0,82(90,0)$ \\
\hline 161725 - Mantém um peso ideal & $0,96(95,0)$ & $0,96(95,0)$ & $0,82(90,0)$ & $0,35(80,0)$ \\
\hline 161726 - Atende às recomendações quanto ao uso de álcool & $0,96(95,0)$ & $0,07(70,0)$ & $0,82(90,0)$ & $0,60(85,0)$ \\
\hline 161727 - Participa de regime para parar de fumar & $1,00(100,0)$ & $0,96(95,0)$ & $0,96(95,0)$ & $0,35(80,0)$ \\
\hline 161728 - Participa em programa de exercício recomendado & $0,96(95,0)$ & $0,82(90,0)$ & $0,96(95,0)$ & $0,60(85,0)$ \\
\hline 161729 - Usa técnicas de conservação de energia & $0,02(65,0)$ & $0,60(85,0)$ & $0,02(65,0)$ & $0,01(60,0)$ \\
\hline 161730 - Equilíbrio entre atividade e repouso & $0,60(85,0)$ & $0,60(85,0)$ & $0,60(85,0)$ & $0,17(75,0)$ \\
\hline 161731 - Realiza rotina normal de vida & $1,00(100,0)$ & $1,00(100,0)$ & $0,96(95,0)$ & $1,00(100,0)$ \\
\hline 161732 - Atende às recomendações quanto à atividade sexual & $0,60(85,0)$ & $0,82(90,0)$ & $0,35(80,0)$ & $0,17(75,0)$ \\
\hline 161733 - Obtém os medicamentos necessários & $0,96(95,0)$ & $0,96(95,0)$ & $0,96(95,0)$ & $0,96(95,0)$ \\
\hline 161734 - Usa medicação conforme prescrição & $1,00(100,0)$ & $0,96(95,0)$ & $1,00(100,0)$ & $1,00(100,0)$ \\
\hline 161735 - Monitora os efeitos terapêuticos dos medicamentos prescritos & $1,00(100,0)$ & $0,60(85,0)$ & $1,00(100,0)$ & $0,35(80,0)$ \\
\hline 161736 - Usa apenas medicação sem receita médica, aprovada pelo profissional de saúde & $0,96(95,0)$ & $0,07(70,0)$ & $0,82(90,0)$ & $0,82(90,0)$ \\
\hline 161737 - Usa técnicas de controle do estresse & $0,96(95,0)$ & $0,96(95,0)$ & $0,82(90,0)$ & $0,35(80,0)$ \\
\hline 161738 - Obtém vacinas contra gripe e pneumonia & $0,82(90,0)$ & $1,00(100,0)$ & $0,60(85,0)$ & $0,60(85,0)$ \\
\hline 161739 - Usa serviços de cuidado de saúde coerentes com a necessidade & $0,96(95,0)$ & $0,96(95,0)$ & $0,96(95,0)$ & $0,96(95,0)$ \\
\hline 161740 - Participa na avaliação do colesterol & $0,60(85,0)$ & $0,35(80,0)$ & $0,60(85,0)$ & $0,60(85,0)$ \\
\hline 161741 - Relata necessidade de assistência financeira & $0,82(90,0)$ & $1,00(100,0)$ & $0,82(90,0)$ & $0,96(95,0)$ \\
\hline 161742 - Mantém as consultas com profissional da saúde & $1,00(100,0)$ & $1,00(100,0)$ & $1,00(100,0)$ & $0,96(95,0)$ \\
\hline 161743 - Mantém plano de emergências médicas & $1,00(100,0)$ & $0,96(95,0)$ & $0,96(95,0)$ & $0,82(90,0)$ \\
\hline 161745 - Adapta a rotina de vida para uma saúde excelente & $0,82(90,0)$ & $0,60(85,0)$ & $0,82(90,0)$ & $0,96(95,0)$ \\
\hline
\end{tabular}

* Teste binomial ** Percentual de acordo sobre a adequação

"Monitora a persistência dos sintomas"; "Monitora a gravidade dos sintomas"; "Relata sinais e sintomas de depressão"; "Monitora a frequência e o ritmo de pulsaçôes" e "Usa técnicas de conservação de energia". O indicador "Usa técnicas de conservação de energia" teve as definiçóes revisadas quanto à relevância.

Dada a impossibilidade de apresentar todas as definiçôes conceituais e operacionais para cada um dos 45 indicadores, os quadros 1 e 2 mostram as definiçôes conceituais e operacionais para os indicadores "Segue a dieta recomendada" e "Monitora a frequência e o ritmo de pulsaçôes" como exemplos do produto final do processo de avaliação.

A seleção desses indicadores para apresentação se deu pela sua relevância na prática clínica e pela possibilidade em mostrar que apesar do valor de $\mathrm{p}$ ter sido sig- 
Quadro 1. Definições conceituais e operacionais do indicador "Segue a dieta recomendada" e versão revisada após a avaliação de enfermeiros especialista e percentual de concordância

\begin{tabular}{|c|c|c|c|c|}
\hline 161720 Atende a dieta recomendada & \multicolumn{2}{|c|}{$\begin{array}{l}\text { Definição operacional da magnitude dos escores da escala } \\
\text { Likert de avaliação }\end{array}$} & \multicolumn{2}{|c|}{$\begin{array}{l}\text { Percentual de } \\
\text { concordância }\end{array}$} \\
\hline \multirow{10}{*}{$\begin{array}{l}\text { Definição conceitual do indicador } \\
\text { Emprega ações que correspondem às orientações para obtenção de dieta saudável. } \\
\text { Definição operacional } \\
\text { Perguntar ao paciente se segue as seguintes recomendações: } \\
\text { - escolhe carnes magras, legumes e vegetais; } \\
\text { - seleciona alimentos livres de gordura e produtos lácteos com baixo teor de gordura } \\
\text { (desnatado); } \\
\text { - minimiza a ingestão de gorduras parcialmente hidrogenadas; } \\
\text { - reduz o volume da dieta e aumenta o } \\
\text { fracionamento ( } 6 \text { a } 8 \text { refeições/dia); } \\
\text { - consome } 50-60 \% \text { do valor energético da dieta; } \\
\text { - consome } 20-30 \text { de fibra e } 150 \text { caloria/grama de proteína; } \\
\text { - não exceder } 30 \% \text { do valor energético da dieta. }\end{array}$} & \multirow{2}{*}{\multicolumn{2}{|c|}{$\begin{array}{l}\text { 1. Não executa qualquer das recomendações para ingestão de } \\
\text { dieta saudável. }\end{array}$}} & $\mathrm{R}$ & 100,0 \\
\hline & & & C & 95,0 \\
\hline & \multirow{2}{*}{\multicolumn{2}{|c|}{$\begin{array}{l}\text { 2. Evita alimentos inadequados na presença de descompensação } \\
\text { ou exacerbação dos sintomas da doença. }\end{array}$}} & $\mathrm{R}$ & 95,0 \\
\hline & & & C & 90,0 \\
\hline & \multirow{2}{*}{\multicolumn{2}{|c|}{$\begin{array}{l}\text { 3. Executa algumas ações apenas na refeição principal e durante } \\
\text { os dias da semana. }\end{array}$}} & $\mathrm{R}$ & 100,0 \\
\hline & & & C & 85,0 \\
\hline & \multirow{2}{*}{\multicolumn{2}{|c|}{$\begin{array}{l}\text { 4. Executa até } 3 \\
\text { ações, até 4x/semana. }\end{array}$}} & $\mathrm{R}$ & 100,0 \\
\hline & & & C & 95,0 \\
\hline & \multirow{2}{*}{\multicolumn{2}{|c|}{ 5. Executa todas as recomendações para dieta saudável. }} & $\mathrm{R}$ & 100,0 \\
\hline & & & C & 90,0 \\
\hline \multicolumn{5}{|l|}{ VERSÃO REVISADA } \\
\hline \multirow{5}{*}{$\begin{array}{l}\text { Definição conceitual do indicador } \\
\text { Emprega ações para obtenção de dieta conforme as orientações do profissional de saúde. } \\
\text { Definição operacional } \\
\text { Perguntar ao paciente se segue as seguintes recomendações: } \\
\text { - escolhe carnes magras, legumes e vegetais; } \\
\text { - seleciona alimentos livres de gordura e produtos lácteos com baixo teor de gordura (desnatado); } \\
\text { - minimiza a ingestão de gorduras parcialmente hidrogenadas; } \\
\text { - reduz o volume da dieta e aumenta o fracionamento (6 a } 8 \text { refeições/dia); } \\
\text { - consome } 50-60 \% \text { do valor energético da dieta na forma de carboidratos; } \\
\text { - consome } 20-30 \mathrm{~g} \text { de fibra; } \\
\text { - consome } 150 \text { caloria/grama de proteína e } 150 \text { caloria/grama de proteína; } \\
\text { - não excede } 30 \% \text { do valor energético da dieta na forma de gordura; } \\
\text { - não excede a quantidade de sódio diária recomendada. }\end{array}$} & & \multicolumn{3}{|c|}{$\begin{array}{l}\text { 1. Não emprega qualquer das recomendações para ingestão de } \\
\text { dieta saudável. }\end{array}$} \\
\hline & & \multicolumn{3}{|c|}{$\begin{array}{l}\text { 2. Emprega ações evitando alimentos inadequados na presença de } \\
\text { descompensação ou exacerbação dos sintomas da doença. }\end{array}$} \\
\hline & & \multicolumn{3}{|c|}{ 3. Emprega algumas ações apenas na refeição principal. } \\
\hline & & \multicolumn{3}{|c|}{$\begin{array}{l}\text { 4. Emprega algumas ações apenas durante os dias úteis da } \\
\text { semana, não atendendo durante os fins de semana. }\end{array}$} \\
\hline & & \multicolumn{3}{|c|}{$\begin{array}{l}\text { 5. Emprega todas as recomendações para dieta saudável } \\
\text { diariamente. }\end{array}$} \\
\hline
\end{tabular}

C - Clareza; R - Relevância

Quadro 2. Definições conceituais e operacionais do indicador "Monitora a frequência e o ritmo de pulsações" e versão revisada após a avaliação dos enfermeiros especialistas e percentual de concordância

\begin{tabular}{|c|c|c|c|}
\hline 161716 Monitora a frequência e o ritmo de pulsações & $\begin{array}{l}\text { Definição operacional da magnitude dos escores da escala } \\
\text { Likert de avaliação }\end{array}$ & \multicolumn{2}{|c|}{ Percentual de concordância } \\
\hline \multirow{10}{*}{$\begin{array}{l}\text { Definição conceitual do indicador } \\
\text { Controla a Frequência Cardíaca (FC) bem como as pulsações } \\
\text { Definição operacional } \\
\text { Verificar se o paciente sabe: } \\
\text { - de quanto deve ser a FC em repouso; } \\
\text { - como verificar pulsações cardíacas; } \\
\text { - a importância da medicação mesmo com a FC normal; } \\
\text { - se reconhece alterações na FC e nas pulsações; } \\
\text { - controla a FC entre } 60 \text { e } 90 \text { bpm em repouso; } \\
\text { - procura manter ritmo sinusal; } \\
\text { - procura o serviço de saúde quando identifica alterações. }\end{array}$} & \multirow{2}{*}{$\begin{array}{l}\text { 1. Não segue qualquer orientação, não conhece as alterações do } \\
\text { ritmo e da FC. }\end{array}$} & $\mathrm{R}$ & 80,0 \\
\hline & & C & 65,0 \\
\hline & \multirow[t]{2}{*}{ 2. Monitoriza apenas a FC. } & R & 70,0 \\
\hline & & C & 70,0 \\
\hline & \multirow{2}{*}{$\begin{array}{l}\text { 3. Monitoriza a FC e o ritmo quando observa exacerbação de } \\
\text { sintomas. }\end{array}$} & R & 80,0 \\
\hline & & C & 75,0 \\
\hline & \multirow[t]{2}{*}{ 4. Monitoriza a FC e o ritmo quando se lembra (1x/semana). } & $\mathrm{R}$ & 75,0 \\
\hline & & C & 70,0 \\
\hline & \multirow{2}{*}{$\begin{array}{l}\text { 5. Segue todas as orientações e conhece as alterações no ritmo } \\
\text { e FC. }\end{array}$} & R & 85,0 \\
\hline & & C & 80,0 \\
\hline \multicolumn{4}{|l|}{ VERSÃO REVISADA } \\
\hline \multirow{5}{*}{$\begin{array}{l}\text { Definição conceitual do indicador } \\
\text { Mensura e reconhece as alteraçõos da Frequência Cardíaca (FC) e do ritmo cardíaco. } \\
\text { Definição operacional } \\
\text { Verificar se o paciente sabe: } \\
\text { - quanto deve ser a FC em repouso; } \\
\text { - verificar as pulsações cardíacas; } \\
\text { - reconhecer alterações na FC e nas pulsações; } \\
\text { - controlar a FC entre } 60 \text { e } 90 \text { bpm em repouso. }\end{array}$} & \multicolumn{3}{|l|}{ 1. Não mensura ou reconhece alterações da FC e do ritmo cardíaco. } \\
\hline & \multicolumn{3}{|c|}{ 2. Reconhece palpitações, mas não reconhece nem mensura frequência e ritmo cardíacos. } \\
\hline & \multicolumn{3}{|c|}{ 3. Reconhece alterações da FC e ritmo cardíaco quando sente "palpitações", mas não os mensura. } \\
\hline & \multicolumn{3}{|c|}{ 4. Reconhece alterações da FC e do ritmo cardíaco quando sente "palpitações" e realiza mensuração. } \\
\hline & \multicolumn{3}{|l|}{ 5. Mensura e reconhece alterações da FC e do ritmo cardíaco. } \\
\hline
\end{tabular}

C-Clareza; R-Relevância

nificativo, as sugestóes feitas pelos especialistas foram acatadas visando ao aprimoramento das definiçôes.

\section{Discussão}

$\mathrm{Na}$ presente investigação, os indicadores de Autocontrole da doença cardíaca foram validados por consenso dos especialistas. Dos 44 indicadores, oito indicadores tiveram as definiçôes operacionais aprimoradas e um indicador teve a definição conceitual aprimorada, visando contribuir para o seu refinamento.

$\mathrm{O}$ uso das linguagens padronizadas de enfermagem no atendimento a pacientes em diferentes ambientes têm sido um desafio para os enfermeiros, e 
os estudos que incentivam e clarificam tais elementos fortalecem essa prática. ${ }^{(9)}$ Assim, o desenvolvimento de definiçóes conceituais e operacionais de um resultado não invalida a utilização de outros resultados, mas contribui para avaliaçôes mais acuradas. ${ }^{(6,7,9)}$

Os indicadores desenvolvidos e validados neste estudo podem ser utilizados para avaliar o comportamento de autocuidado antes e após a implementação de intervençôes, auxiliando os enfermeiros a selecionar intervençóes apropriadas com maior impacto para motivar os pacientes e enfermeiros a buscarem constantemente o controle da doença. Os enfermeiros podem selecionar um grupo de indicadores adequados a um determinado paciente, estimando uma meta, que pode ser avaliada por um período de tempo. A avaliação seriada em um programa de acompanhamento pode ser realizada com indicadores precisos para cada estratégia implementada pelo profissional. Portanto, mesmo que o resultado NOC tenha uma quantidade estimável de indicadores, eles podem ser selecionados de acordo com a necessidade. ${ }^{(5)}$

Consideram-se vários indicadores indispensáveis para o controle da doença cardíaca, especificamente aqui, para o indivíduo com IC. Destacam-se os indicadores de monitoramento do surgimento, persistência, gravidade e frequência dos sintomas; a identificação de sinais de piora da doença; obtenção de cuidados de saúde frente à ocorrência de sinais de alerta; a limitação de ingestão de sódio; o monitoramento do peso corporal; a utilização de medicamentos conforme prescrição e a obtenção de vacina de pneumonia e gripe.

Os indicadores do Sistema de Classificação de Enfermagem como a NOC, requerem melhor especificação e descrição com adequado rigor científico e menor subjetividade. ${ }^{(9)} \mathrm{O}$ desenvolvimento de definiçôes auxilia a estabelecer o grau de melhora ou piora do paciente, proporcionando uma mensuração mais acurada dos escores dos indicadores selecionados, tornando-os mais sensíveis e representativos das respostas dos pacientes às intervençôes e menos subjetiva, melhorando a utilização da taxonomia na prática clínica. ${ }^{(7,29)}$

A legitimação dos elementos NOC tem sido indicada em diferentes estudos que objetivaram elaborar e validar definiçóes de indicadores de resultados, tornando-os menos subjetivos para as avaliaçôes. ${ }^{(6,9,10,29)} \mathrm{O}$ desenvolvimento de definiçóes conceituais e operacionais é essencial neste processo e contribui para a reprodutibilidade das investigaçôes e redução da inconsistência das análises dos enfermeiros. ${ }^{(10)}$

Como muitos indicadores não são minuciosamente descritos na literatura, a avaliação crítica dos enfermeiros especialistas é fundamental para o aperfeiçoamento das definiçóes. Definiçōes construídas para esses indicadores são frequentemente baseadas nas consideraçóes do pesquisador e na extensa revisão científica do assunto e da literatura relacionada. Esse problema é intensificado ao se estabelecer definiçôes dos itens da escala Likert, pois a diferenciação entre os níveis deve refletir melhora ou piora dos pacientes em relação a um resultado esperado. O estabelecimento de um nível de estado/ comportamento do paciente frente a um resultado é obrigatório, mas, na ausência de definiçôes podem não ser claras e dificultar o registro de informaçôes bem como o uso da escala na indicação do nível de gravidade. ${ }^{(5)}$

Um estudo realizado por um grupo de enfermeiros que buscava obter consenso na seleção de diagnósticos da NANDA-I, intervençôes NIC e resultados NOC, para o cuidado à pacientes com IC em domicílio, relatou 6 diagnósticos, 11 intervençóes e 7 resultados. Os resultados propostos foram: Tolerância à atividade; Conservação de energia; Conhecimento: regime de tratamento; Controle dos Sintomas; Conhecimento: Medicação; Equilíbrio hídrico e Participação familiar no cuidado profissional. ${ }^{(19)} \mathrm{O}$ resultado "Autocontrole da doença cardíaca" contém indicadores que englobam todos os resultados mencionados com exceção do resultado "Participação familiar no cuidado profissional". Nesse contexto, deve ser ponderado sobre o que é mais apropriado para a Ciência da Enfermagem e para a prática clínica de enfermagem: um resultado amplo ou resultados mais específicos.

Um estudo analisou 101 artigos descrevendo o autocontrole em indivíduos com doenças crônicas, definindo-o como um processo diário dinâmico e interativo de engajamento para o controle da doença. 
Este estudo identificou três categorias no processo de autocontrole: 1-focando nas necessidades da doença; 2-ativando recursos; e 3-vivendo com a doença crônica. Para cada categoria, tarefas e habilidades foram delineadas para se obter estratégias para o controle da doença. ${ }^{(30)}$ Os indicadores do resultado em estudo também podem ser considerados tarefas e habilidades requeridas pelo indivíduo para controlar a IC, com a vantagem de estarem acompanhados de escala de avaliação e a descrição para cada escore.

Apesar das tentativas de buscar o maior número de especialistas possível, obteve-se a participação de 20, que pode ser considerado um número pequeno, constituindo possível limitação deste estudo. Foi prevista apenas uma abordagem para avaliação das definiçóes pelos enfermeiros, que não foram novamente consultados para exame da versão revisada, o que também pode ser considerada limitação. É possível que novas rodadas pudessem contribuir com um maior grau de refinamento das definições, no entanto, julga-se que os resultados obtidos constituem importante ponto de partida para novas pesquisas e estudos de validação clínica do resultado de enfermagem "Autocontrole da doença cardíaca".

\section{Conclusão}

Definiçóes conceituais e operacionais para os indicadores do resultado NOC "Autocontrole da doença cardíaca" foram estabelecidas e consensualmente validadas por um grupo de especialistas. A maioria das definiçóes foi considerada clara e relevante. Apenas uma das definiçôes não foi considerada relevante, porque tais informaçóes poderiam ser obtidas de outro indicador. Algumas definições foram refinadas para atender as sugestôes feitas pelos especialistas, tornando-as mais claras e facilitando o processo de avaliação clínica. $\mathrm{O}$ resultado $\mathrm{NOC}$ e seus indicadores podem ajudar os profissionais a planejar o atendimento e fornecer informações úteis sobre os tópicos necessários à avaliação dos indivíduos com a doença. Portanto, os pesquisadores devem ser fortemente encorajados a desenvolver definiçôes para indicadores de fácil aplicabilidade. Uma vez estabelecidas as definiçóes conceituais e operacionais dos indicadores, o processo de avaliação e o acompanhamento tornam-se mais acessíveis e confiáveis, mesmo quando realizados por diferentes avaliadores, o que diminui equívocos e orienta a prática clínica para melhores resultados.

\section{Agradecimentos}

Ao Conselho Nacional de Ciência e Tecnologia (CNPq); bolsa de produtividade em pesquisa nível 1B para Alba Lúcia Bottura Leite de Barros. À Coordenação de Aperfeiçoamento de Pessoal de Nível Superior (CAPES); bolsa de doutorado para Agueda Maria Ruiz Zimmer Cavalcante.

\section{Colaborações}

Cavalcante AMRZ, Lopes CT, Swanson E, Moorhead AS, Bachion MM e Barros ALBL declaram que contribuíram com a concepção do estudo, análise e interpretação dos dados, redação do artigo, revisão crítica relevante do conteúdo intelectual e aprovação da versão final a ser publicada.

\section{Referências}

1. Arries EJ. Patient safety and quality in healthcare: nursing ethics for ethics quality. Nurs Ethics. 2014;21(1):3-5.

2. Cañón-Montañez W, Oróstegui-Arenas M. [Reliability of nursing outcomes classification label "Knowledge: cardiac disease management (1830)" in outpatients with heart failure]. Enferm Clin. $2015 \mathrm{Jul} ; 25(4): 186-97$.

3. Müller-Staub M, Lavin MA, Needham I, van Achterberg T. Nursing diagnoses, interventions and outcomes - application and impact on nursing practice: systematic review. J Adv Nurs. 2006;56(5):514-31.

4. Carvalho EC, Cruz DA, Herdman TH. Contribution of standardized languages for knowledge production, clinical reasoning and clinical Nursing practice. Rev Bras Enferm. 2013;66(Esp):134-41.

5. Moorhead S, Johnson M, Mass M, Swanson E, editors. Nursing Outcomes Classification (NOC). 5th ed. MO: Mosby; 2013.

6. Oliveira AR, de Araujo TL, de Carvalho EC, Costa AG, Cavalcante TF, Lopes MV. Construction and validation of indicators and respective definitions for the nursing outcome Swallowing Status. Rev Lat Am Enfermagem. 2015;23(3):450-7.

7. Vitor AF, Araújo TL. Definições para o resultado de enfermagem comportamento de prevenção de quedas: uma revisão integrativa. Rev Eletr Enf. 2011;13(2):313-22. 
8. de Sousa VE, Lopes MV, da Silva VM, Keenan GM. Defining the key clinical indicators for ineffective breathing pattern in paediatric patients: a meta-analysis of accuracy studies. J Clin Nurs. 2015;24(1314):1773-83

9. da Silva VM, Lopes MV, de Araujo TL, Beltrão BA, Monteiro FP, Cavalcante TF, et al. Operational definitions of outcome indicators related to ineffective breathing patterns in children with congenital heart disease. Heart Lung. 2011;40(3):e70-7.

10. Chantal Magalhães da Silva N, de Souza Oliveira-Kumakura AR, Moorhead S, Pace AE, Campos de Carvalho E. Clinical Validation of the Indicators and Definitions of the Nursing Outcome "Tissue Integrity: Skin and Mucous Membranes" in People With Diabetes Mellitus. Int J Nurs Knowl. 2017;28(4):165-70.

11. Thompson DR, Ski CF. Patient-reported outcome measures in cardiovascular nursing. Eur J Cardiovasc Nurs. 2015;14(5):370-1.

12. Rohde LE, Montera MW, Bocchi EA, Clausell NO, Albuquerque DC, Rassi S, et al.; Comitê Coordenador da Diretriz de Insuficiência Cardíaca. Diretriz Brasileira de Insuficiência Cardíaca Crônica e Aguda. Arq Bras Cardiol. 2018;111(3):436-539.

13. Brasil. Ministério da Saúde. Sistema de Informações Hospitalares do SUS. Informações de saúde. Brasília (DF): Ministério da Saúde; 2016. [citado 2019 Jun20]. Disponível em: http://tabnet.datasus.gov.br/cgi/ tabcgi.exe?sih/cnv/niuf.def.

14. Ambrosy AP, Fonarow GC, Butler J, Chioncel 0, Greene SJ, Vaduganathan $\mathrm{M}$, et al. The global health and economic burden of hospitalizations for heart failure: lessons learned from hospitalized heart failure registries. J Am Coll Cardiol. 2014;63(12):1123-33.

15. Jonkman NH, Westland H, Groenwold RHH, Agreen S, Atienza F, Blue $\mathrm{L}$, et al. Do Self-Management Interventions work in patients with Heart Failure? An individual patient data meta-analysis. Circulation. 2016;133(120):1189-98

16. Smeulders ES, van Haastregt JC, Ambergen T, Janssen-Boyne JJ, van Eijk JT, Kempen Gl. The impact of a self-management group programme on health behaviour and healthcare utilization among congestive heart failure patients. Eur J Heart Fail. 2009;11(6):609-16.

17. Zampieron A, Aldo S, Corso M. A retrospective study of nursing diagnoses, outcomes, and interventions for patients admitted to a cardiology rehabilitation unit. Int J Nurs Terminol Classif. 2011 0ct;22(4):148-56.

18. Carneiro CS, Oliveira AP, Lopes JL, Bachion MM, Herdman TH, Moorhead SA, et al. Outpatient clinic for health education: contribution to self-management and self-care for people with heart failure. Int $\mathrm{J}$ Nurs Knowl. 2016;27(1):49-55.
19. Azzolin K, Mussi CM, Ruschel KB, de Souza EN, de Fátima Lucena A, Rabelo-Silva ER. Effectiveness of nursing interventions in heart failure patients in home care using NANDA-I, NIC, and NOC. Appl Nurs Res. 2013;26(4):239-44.

20. Riegel B, Carlson B, Moser DK, Sebern M, Hicks FD, Roland V. Psychometric testing of the self-care of heart failure index. J Card Fail. 2004;10(4):350-60.

21. Jaarsma T, Strömberg A, Mårtensson J, Dracup K. Development and testing of the European Heart Failure Self-Care Behaviour Scale. Eur J Heart Fail. 2003;5(3):363-70.

22. Bocchi EA, Marcondes-Braga FG, Ayub-Ferreira SM, Rohde LE, Oliveira WA, Almeida DR, et al. Sociedade Brasileira de Cardiologia. III Diretriz Brasileira de Insuficiência Cardíaca Crônica. Arq Bras Cardiol. 2009;93(1 Supl.1):1-71.

23. Riegel B, Moser DK, Anker SD, Appel LJ, Dunbar SB, Grady KL, et al.; American Heart Association Council on Cardiovascular Nursing; American Heart Association Council on Cardiovascular Nursing; American Heart Association Council on Clinical Cardiology; American HeartAssociation Council on Nutrition, Physical Activity, and Metabolism; American Heart Association Interdisciplinary Council on Quality of Care and Outcomes Research. State of the science: promoting self-care in persons with heart failure: a scientific statement from the American Heart Association. Circulation. 2009;120(12):1141-63.

24. Lopes MV, Silva VM, Araújo TL. Methods for establishing the accuracy of clinical indicators in predicting nursing diagnoses. Int J Nurs Knowl. 2012;23(3):134-9.

25. Cavalcante AM, Lopes CT, Brunori EH, Swanson E, Moorhead SA, Bachion MM, et al. Self-care behaviors in heart failure. Int J Nurs Knowl. 2018;29(3):146-155

26. Martins GA, Pelissaro J. Sobre conceitos, definições e constructos nas ciências contáveis. Rev Adm Contabil Unisinos. 2005;2(2):78-84.

27. Grant JS, Kinney MR. Clinical referents for nursing diagnoses. J Neurosci Nurs. 1992;24(2):94-8.

28. Alexandre NM, Coluci MZ. [Content validity in the development and adaptation processes of measurement instruments]. Cien Saude Colet. 2011;16(7):3061-8. Review. Portuguese.

29. Seganfredo DH, Almeida MA. Nursing outcomes content validation according to Nursing Outcomes Classification (NOC) for clinical, surgical and critical patients. Rev Lat Am Enfermagem. 2011;19(1):34-41.

30. Schulman-Green D, Jaser S, Martin F, Alonzo A, Grey M, McCorkle R, et al. Processes of self-management in chronic illness. J Nurs Scholarsh. 2012;44(2):136-44. 